\title{
Exploring the Feasibility and Acceptability of a Home-based Smart Health Sensing System to Improve Cancer Pain Management for Patients and Family Caregivers
}

\section{益UVAENGINEERING}

LINK LAB

\section{BACKGROUND}

- Cancer pain is a significant problem; affects $60-90 \%$ of patients with cancer

- Most cancer pain management occurs at home and family caregivers play a large role

- Opioids are important tool to manage serious cancer pain, but need careful monitoring

- Smart Health technology can support safe and effective management of cancer pain in the home environment

\section{PURPOSE}

- Pilot test Behavioral and Environmental Sensing and Intervention for $\underline{\text { Cancer }}$ (BESI-C) to:

1) Understand contextual factors that predict and influence the experience of breakthrough pain

2) Explore the dyadic effect between patient and caregiver experiences related to cancer pain

\section{BESI-C SYSTEM COMPONENTS}

Environmental Sensors

Ambient audio

Barometric pressure

Light

- Temperature

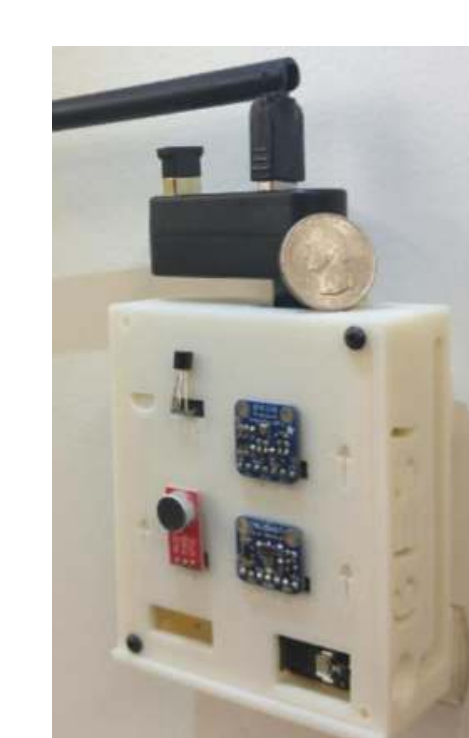

\section{Estimote Beacons}

Localization within home

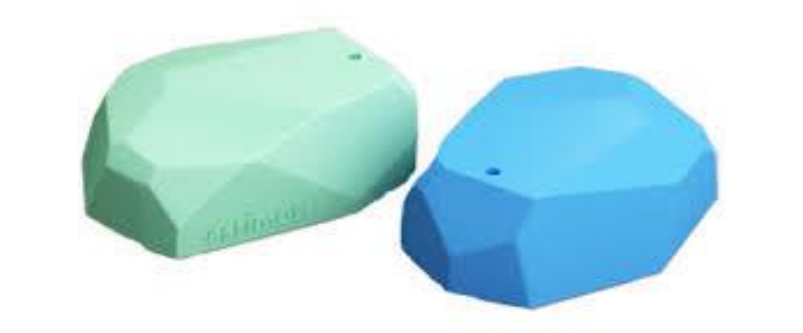

Smart Watch

- Heart rate

- Accelerometer

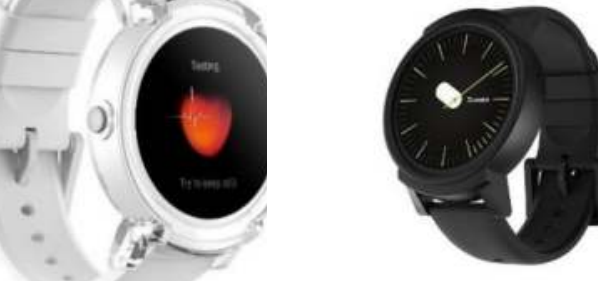

- Ecological Momentary Assessments (EMAs)

\section{BESI-C SYSTEM ARCHITECTURE}
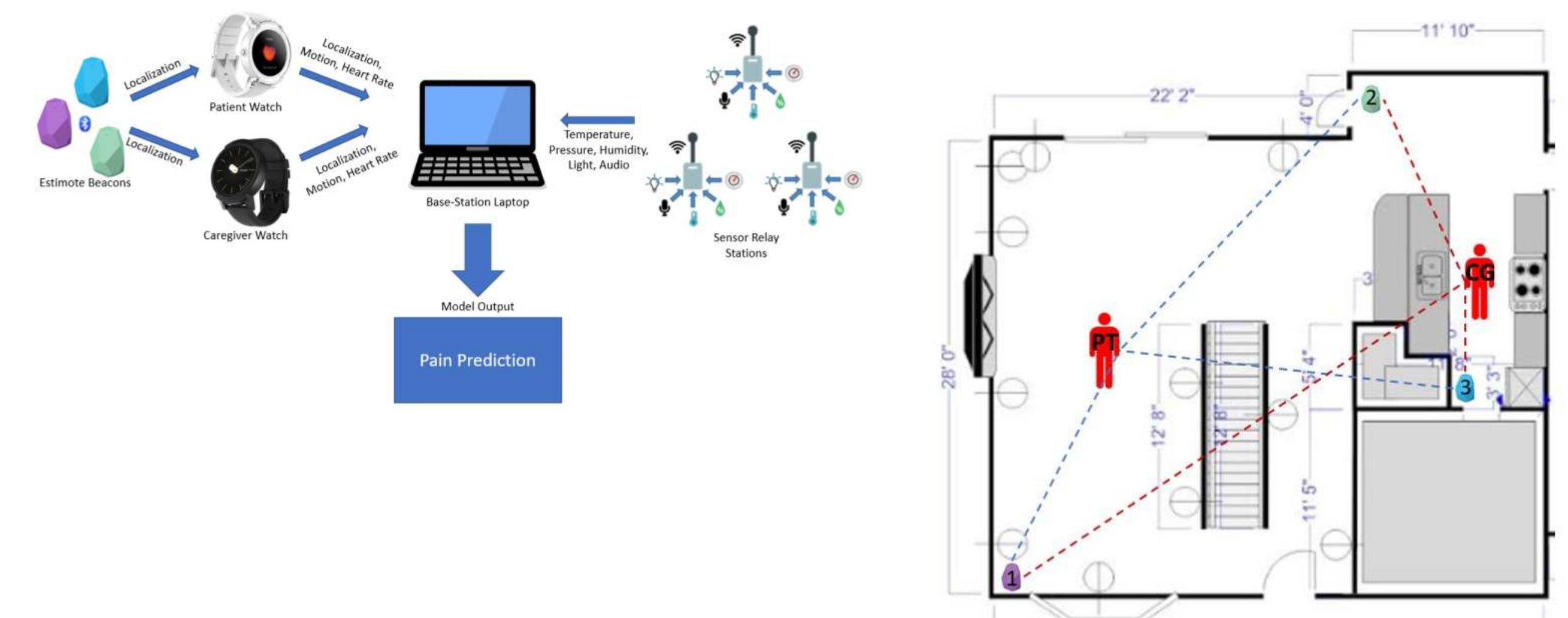

\section{SMART WATCH 'APP'}

- EMAs mark pain events and assess overall quality of life

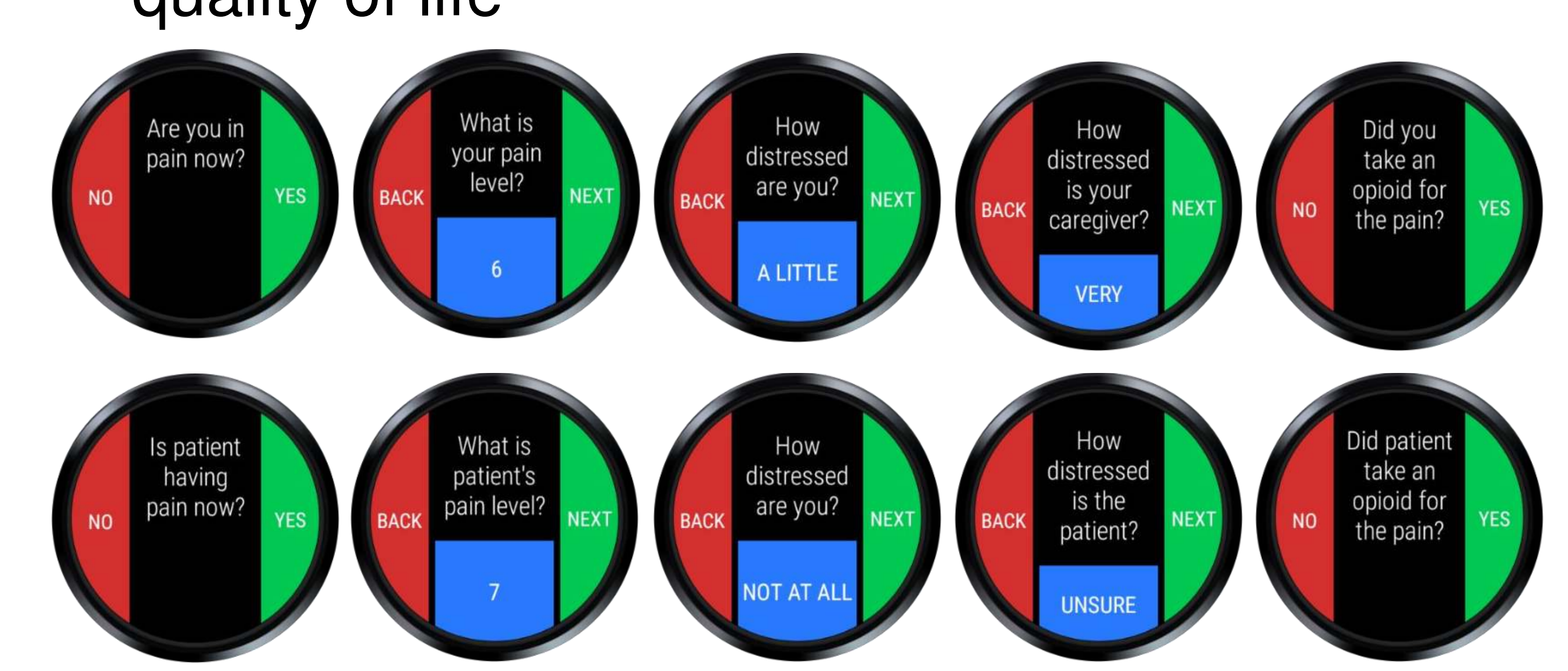

Virginia LeBaron', Leslie Blackhall², Ridwan Alam³, James Hayes ${ }^{3}$, Nutta Homdee, Kate Gordon', Yudel Martinez ${ }^{3}$, Emmanuel Ogunjirin' ${ }^{3}$, Seth VandeBraak ${ }^{3}$ Randy Jones', Tanya Thomas', John Lach

University of Virginia (UVA) School of Nursing; ${ }^{2}$ UVA School of Medicine; ${ }^{3}$ UVA School of Engineering and Applied Science

\section{STUDY DESIGN}

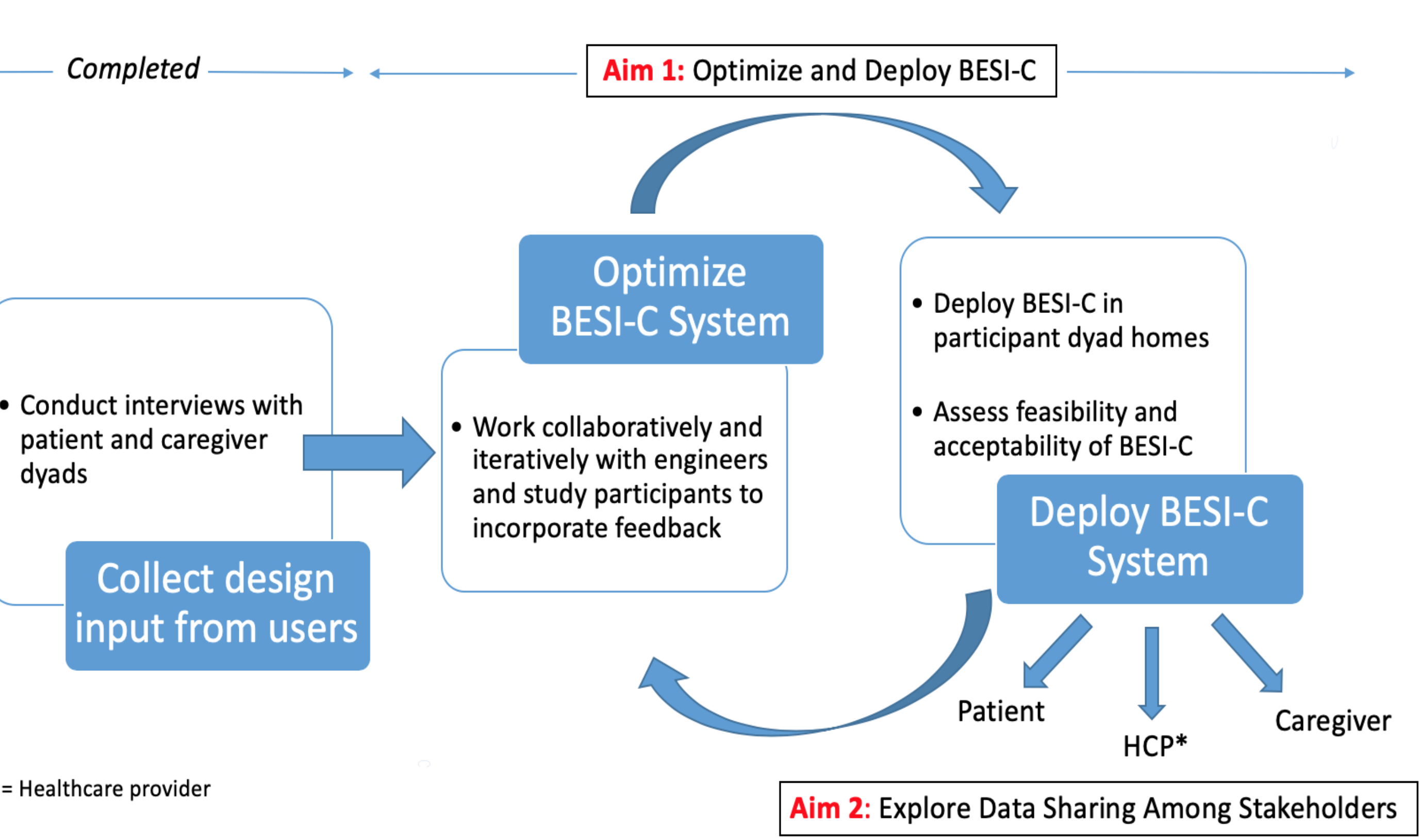

METHODS

Sample:

- Recruited from UVA Oncology Palliative

Care Clinic

- Patients with advanced cancer and primary family caregiver

- Prescribed opioids for cancer related pain

- Pain scores of $\geq 6$

Phase I: Qualitative Interviews (completed)

- 10 dyads; structured interviewed to assess:

1) Challenges of managing cancer pain at home

2) Contextual variables relevant to cancer pain

3) BESI-C design feedback

Phase II: BESI-C deployments (in progress)

- 15 dyads (target); in-home pilot testing to assess:

1) Fidelity of data capture

2) System utilization

3) Dyad experiences with BESI-C system

\section{RESULTS}

Phase I: Qualitative Interviews

Theme 1. Cancer pain is unpredictable, stressful and impacts daily life, especially sleep and social interactions

- It hits me so bad sometimes it brings tears to my eyes... When I'm in really, really bad pain it gets me down. I get depressed and it's like, 'God, is this ever gonna quit?' - Pt 10

- We went from being outside every day and doing things to pretty much watch watching her lay on the couch. - CG 9

Theme 2. Difficulty tracking medications; fears of running out of medication; balancing side effects

- I worry about the medications and if I'm gonna have enough or God forbid if I lose some. - Pt 10

- Most difficult? Taking my medicine. Sometimes I'll take more than I should if I'm really in a lot of pain, and I know l'm not supposed to but it's hard not to. - Pt 2

- Keeping up with the medications and as they change. - CG 5

\section{RESULTS (CONTINUED)}

Phase I: Qualitative Interviews

Theme 3. Dyads validated proposed variables to measure by BESI-C; open to testing system

- I think it's a good idea myself. I think that would make a big difference in monitoring some things. - CG 6

- Seems interesting...I think that I would try it. - Pt 5

- As long as it ain't watching us! - CG 9
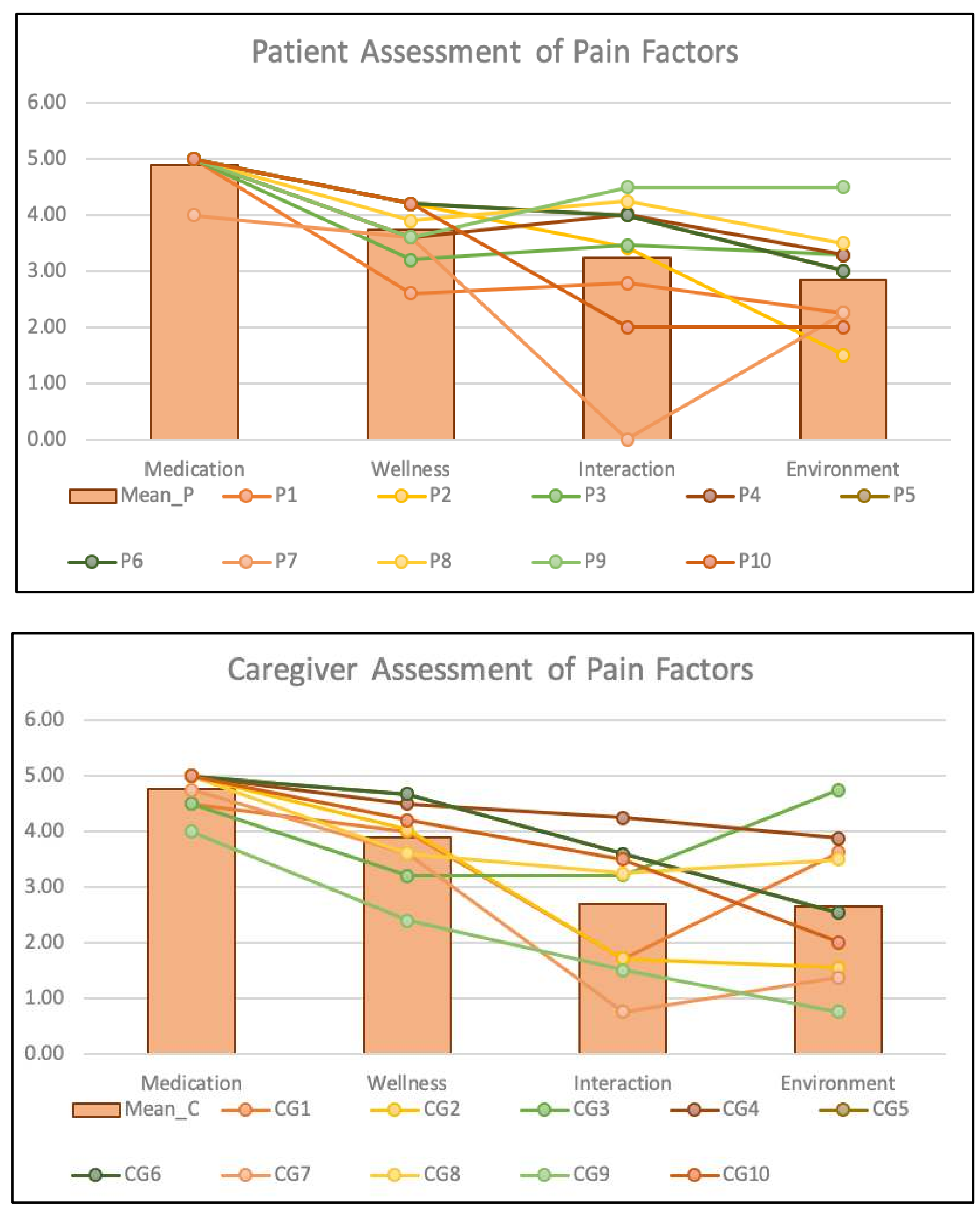

Phase II: In-Home Deployments

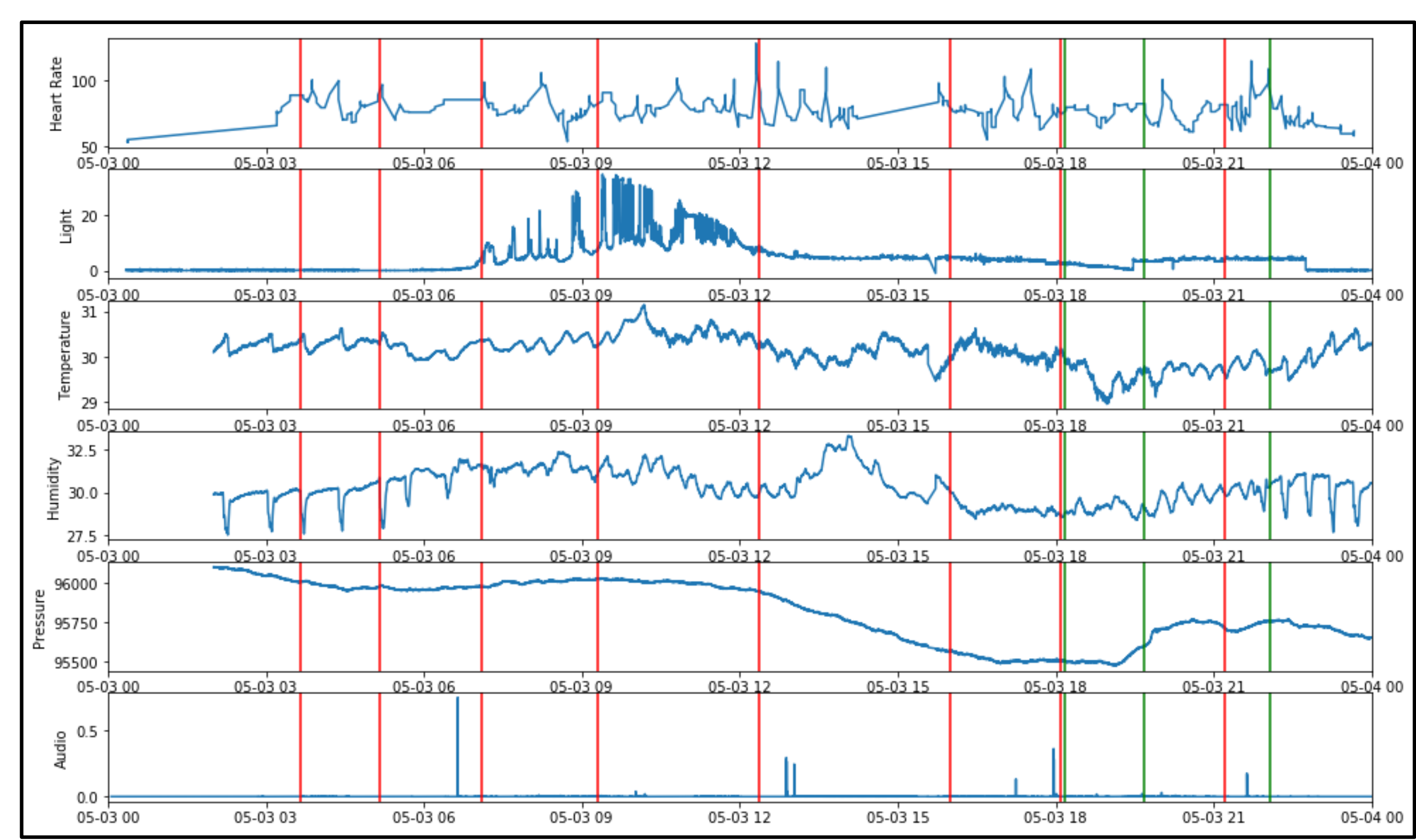

- Preliminary data: Dyad marked pain events, environmental data from the living room, and patient heart rate data

\section{CONCLUSIONS}

- Dyads are open to novel approaches to managing pain in the home

- Significant potential exists for BESI-C to inform and deliver personalized pain interventions

- Future work to deploy BESI-C in rural areas and home hospice programs 